\title{
Elementos de una organización educativa inclusiva para la respuesta a alumnado con necesidades educativas especiales de educación secundaria en Italia. La situación en un instituto profesional
}

\author{
Luisadora PASCALE ${ }^{1}$, María del Mar FERNÁNDEZ MARTÍNEZ ${ }^{2}$ \\ y José Juan CARRIÓN MARTÍNEZ ${ }^{3}$ \\ ${ }^{1}$ Instituto de Educación Superior Bonghi-Rosmini-Lucera, Italia \\ ${ }^{2}$ Departamento de Psicología, Universidad de Almería \\ ${ }^{3}$ Departamento de Educación, Universidad de Almería
}

(Recibido el 17 de marzo de 2018; Aceptado el 25 de Julio de 2018)

\begin{abstract}
RESUMEN El presente trabajo aborda la experiencia de la inclusión escolar en su dimensión organizacional en la etapa de educación secundaria obligatoria en el contexto italiano. Aun siendo un tema recurrente, no deja de ser un tema que en la práctica preocupa con intensidad al profesorado, a las familias y a los gestores educativos, por ello se ha tenido como objetivo fundamental analizar la situación real de la inclusión vivida y percibida por los actores sobre la organización de su institución. Metodológicamente se ha afrontado desde un estudio de caso de un instituto de secundaria de relevancia con respecto a la problemática, por su tamaño, por el impacto del problema en su seno y por su ubicación en una zona de sur de Italia con especial penetración social y escolar de la problemática de la diversidad en los centros escolares. Nos hemos encontrado con unos resultados que denotan la gran fractura que existe entre la evolución del discurso y su proyección real. Mientras la inicial integración ha quedado subsumida desde hace ya tiempo, en un paradigma más universal como es el de la inclusión, la realidad arroja datos que nos retrotraen a momentos menos evolucionados de este paradigma. La inclusión es más teórica que real, alcanza el plano de la planificación y del diseño, pero menos el de la práctica docente, con déficit notables en coordinación, en trabajo de equipo específico, y con una demanda de formación reiterada.
\end{abstract}

Palabras clave: inclusión escolar, necesidades especificas de apoyo educativo, diversidad, organización educativa, diversidad

\section{Elements of the inclusive school organization for the response to students with special needs in secondary education in Italy. The situation in a professional secondary schools}

\begin{abstract}
This paper addresses the experience of school inclusion in the organizational dimension in the stage of compulsory secondary education in the Italian context. Even as a recurring theme, in practice intensely concerned about the professed, families and educational managers, therefore it has as main objective to analyze the actual situation of inclusion lived and perceived by the actors on organization of your institution. Methodologically, it has been addressed from a case study of a secondary school of
\end{abstract}


relevance to the issue, by size, by the impact of the problem on its breast and its location in an area of southern Italy special social penetration and school of the problem of diversity in schools.

We have found some results that denote the great fracture that exists between the evolution of discourse and its real projection. While the initial integration has been subsumed for a long time, in a more universal paradigm such as inclusion, the reality throws data that takes us back to less evolved moments of this paradigm.

Inclusion is more theoretical than real, reaches the level of planning and design, but less the plane the teaching practice, with significant deficits in coordination, in specific team work, specific equipment, repeatedly with a demand for training.

Key words: school inclusion, special needs, diversity, educational organization, diversity

Correspondencia: José Juan Carrión Martínez. Departamento de Educación, Universidad de Almería. jcarrion@ual.es

\section{Introducción}

La atención educativa al alumnado con necesidades educativas especiales, no es un problema nuevo (Ainscow, 2004; Barton, 1988, 2008; Barton y Armstrong, 2001; Carrión Martinez, 2001). Ya desde hace décadas se ha venido abordando esta cuestión, tanto desde la perspectiva reflexiva, como desde de la investigación empírica, pero tan reiterado como vigente podemos considerar el problema (Sandri, 2014). Es una problemática que se reinventa constantemente a sí misma, tanto en la conceptualización estructural de la situación del sujeto: deficiencia, minusvalía, discapacidad, diversidad funcional, como desde la respuesta educativa: sistema diferenciado y segregado, integración escolar, etc. hasta llegar, en una revisión constante, al más avanzado modelo de inclusión.

En concreto en este trabajo abordamos aspectos de la cuestión de la gestión y organización de la inclusion educativa, percibida, vivida y sentida fundamentalmente por el profesorado y la familia en la educación secundaria. Para ello nos hemos acercado al interior de un instituto de educacion secundaria y profesional del sur de Italia.

\section{Integración/inclusión, caras de un mismo proceso}

Según D’Alesssio (2011a, 2011b, 2014, 2015) el concepto de inclusión apunta a un objetivo muy elevado, a saber, mejorar la sociedad eliminando todas las formas de discriminación, empezando por el sistema escolar, donde existen tales formas y, promoviendo por lo tanto, un sistema totalmente reformado en sus aspectos estructurales y pedagógicos para superar todas las formas de diferencia entre los estudiantes. Una nueva escuela, lejos de la idea de necesidades educativas especiales (en adelante, NEE) o integración, que busque superar la dicotomía integración/inclusión. Hemos de acercarnos a una escuela abierta que pueda incluir todas las individualidades de los estudiantes, diferentes entre sí, como un derecho a disfrutar de una participación justa y al aprendizaje (Ianes, 2009; Ianes, Demo, y Zambotti, 2010).

Dovigo (2007) argumenta que los estudiantes desfavorecidos, con desventajas socioeconómicas, etc. constituyen una parte muy relevante de la clase, pudiendo los llamados alumnos normales llegar a representar una minoría.

Si se piensa en la diversidad del alumnado, emergen cuáles son sus necesidades y, por lo tanto, se puede transformar la dificultad en un recurso. A menudo, se confía el alumno con 
discapacidad, con NEE, etc. al maestro de apoyo, sin embargo, desde la perspectiva de que todos los estudiantes tienen sus propias necesidades individuales, todos necesitarían apoyo durante su escolarización. En consecuencia, cada alumno es una persona que debe ser entendida, comprendida e interpretada en sus diferentes características, única y diferente de todas las demás (Sandri, 2014).

Una escuela abierta a todos debe tener en cuenta la diversidad del alumnado y estas diferencias necesitan ser entendidas por los docentes escuchando, interpretando y respondiendo con diferentes herramientas adaptadas a las diversas individualidades. Una escuela que flexibiliza sus modelos organizativos con relación a las peculiaridades y los diferentes tipos de alumnado es una escuela de calidad que trabaja en interés de todos los alumnos (Dovigo, 2007; Hierro Parolin, 2010; Echeíta Sarrionandía y Duk Homad, 2008; Echeíta Sarrionandía et al., 2009; Toboso Martín et al., 2012; Pascale, Carrión-Martínez y Fernández-Martínez, 2017).

\section{Fundamentos de una gestión escolar inclusiva}

Hoy en la escuela se habla no sólo de la integración de la persona con discapacidad, sino de la integración de las personas de diferentes culturas y países, los niños con dificultades ambientales, los chicos y chicas absentistas, etc. (Sandrone, 2010). Por lo tanto, un docente que tenga una diversidad de alumnado con diferentes necesidades educativas derivadas de diferentes problemas (comportamiento, relaciones familiares difíciles, poco interés en la escuela, problemas socioeconómicos, etc.), tendrá que seleccionar, tomar decisiones y desarrollar prácticas organizacionales y didácticas individualizadas. Así que esta nueva óptica pedagógica ha anulado por completo la antigua visión integradora (d'Alonzo, 2008).

Las diversas necesidades dentro del aula deben ser aceptadas y valoradas, ya que cada individualidad se considerará un estímulo e incentivo para toda la clase. En este contexto, la escuela se debe convertir en un entorno estimulante para todos (d'Alonzo, 2008; Gelati, 2004; Avilés Soler, Fernández Martínez, Hernández Garre, Luque de la Rosa y Gutiérrez Cáceres, 2017). Según d'Alonzo $(2008,2012)$ algunos aspectos relevantes para que podamos hablar de un proceso abierto a la gestión escolar inclusiva son referir la planificación educativa a las necesidades y no a los límites, promover un diseño basado en sus fortalezas, sus recursos y capacidades, así como prestar atención al alumnado creando una atmósfera de recepción positiva y relacional.

Como apunta Maviglia (2009), cuando un docente programa, debe dotarse de una cierta flexibilidad, que debe tener como punto de partida la singularidad y los estados de ánimo cambiantes de la relación y el aprendizaje de sus alumnos, más allá de si existe o no la presencia de un alumno con discapacidad o un docente de apoyo.

\section{MÉTODO}

\section{Objetivos}

Analizar las estrategias organizativas inclusivas que la escuela secundaria italiana implementa en la atención al alumnado con necesidades específicas de apoyo educativo (en adelante, ACNEAE). 


\section{Enfoque metodológico}

Hemos llevado a cabo una metodología de tipo cualitativo, naturalista e interpretativa (Albert Gómez, 2007; Goetz y LeCompte, 2010; Flickr, 2014; Delli Zotti, 2001; Lucisano y Salerni, 2002; Cecconi, 2002). El método utilizado has sido el estudio de caso (Yin, 2014; Stake, 2010; Sturman, 1998)

\section{Contexto e informantes}

El contexto es el italiano, donde el $\mathrm{ACNEE}^{1}$ está incluido en todas las escuelas y legalmente reconocido como un recurso para el crecimiento y el desarrollo socioeducativo de todos (Sandri, 2014). Se ha abordado el caso de una escuela secundaria profesional, que denominaremos para preservar los compromisos éticos negociados Istituto professionale Odeon, ubicado en la provincia de Foggia (Italia). El criterio por el que se eligió este centro fue su tamaño, con un número considerable de ACNEE y un número igualmente grande de personal docente (profesores adjuntos, profesores titulares, educadores) que participan en él, siendo un centro muy involucrado en el problema objeto de estudio.

Tabla 1. Tipo de informantes, $n^{\circ}$ de entrevistas y claves de identificación

\begin{tabular}{|c|c|c|c|}
\hline $\begin{array}{c}\text { Tipo } \\
\text { de informante }\end{array}$ & $\begin{array}{c}\mathrm{N}^{\mathrm{o}} \text { sujetos } \\
\text { Informantes/participantes }\end{array}$ & $\begin{array}{l}\text { Código de } \\
\text { informante }\end{array}$ & $\begin{array}{l}\text { Código } \\
\text { de técnica }\end{array}$ \\
\hline Profesor de apoyo & 6 & DOSOS & Entrevista. \\
\hline Personal técnico de apoyo & 1 & RESOS & $\operatorname{En} 2$ \\
\hline Profesor curricular & 4 & DOCUR & $\begin{array}{l}\text { Observacion: } \\
\text { On }\end{array}$ \\
\hline Vicedirector & 2 & VIDIR & \\
\hline Orientador & 2 & FACIL & Análisis de \\
\hline Director & 1 & DIRSC & Documentos: \\
\hline Coordinador & 1 & DIRDI & $\mathrm{AD}$ \\
\hline Familiar & 1 & GENIT & \\
\hline Total informantes/participantes & 18 & & \\
\hline Total de entrevistas & 18 & & \\
\hline Total sesiones de observación & 28 & & \\
\hline Documentos analizados & 1 & & \\
\hline
\end{tabular}

Con respecto a los informantes la atención se centró en el personal docente, la familia de los ACNEE y los directivos escolares. Cada entrevistado ha sido identificado por su clave de informante precedida de dos dígitos alfanuméricos que identifican la técnica empleada y la secuencia ordinal que esta ocupa (Tabla 1).

1 Usamos la expresión en español Alumnado con Necesidad Específica de Apoyo Educativo (ACNEE), como interpretación contextualizada de la expresión italiana BES (Bisogni Educativi Speciale).

2 (n) Identifica el número de la acción de recogida de información ya sea en entrevista, ya en observación. código de informante.

(C) Psy, Soc, \& Educ, 2019, Vol. 11(1) 


\title{
Técnicas
}

Tal y como indicaban Tortora y Santini (2001) o Lanzara (1988) para la recopilación de datos se utilizaron las técnicas de la entrevista y la observación.

Con respecto a la observación indicar que la hemos utilizado para la búsqueda de información relevante, permitiendonos acercarnos al pensamiento, motivaciones, creencias, así como a elementos no verbales (Tejada Fernández, 1997; Taylor y Bogdan, 2010; Goetz y LeCompte, 2010; Valdez Contreras, 2016). Las observaciones involucraron 3 clases.

Para catalogar y preservar el anonimato, se utilizan las claves asignadas a los tipos de participantes en la Tabla 1.

El análisis de documentos (McKernan, 1999, citado en Valdez Contreras, 2016; Santos Guerra, 2008; Stake, 2010) se ha utilizado en los documentos organizacionales, como en el caso de los centros educativos italianos el Piano dell'Offerta Formativa ${ }^{3}$ (en adelante, POF).

\section{Precategorías}

Las categorías indagativas fueron las que se recogen en la Figura 1.

\section{PRECATEGORÍAS}

\author{
Planificación y procesos de organización \\ Inclusión en la vertiente didáctica \\ Coordinación \\ Formación y recursos \\ Colaboración institucional
}

Figura 1. Relación de precategorías

\section{Acceso al campo}

Se desarrolló en dos etapas (Figura 2). Inicialmente, en la primera fase, la fase preparatoria, se trabajó para establecer contactos con el Director del Instituto. Posteriormente se dió paso a la fase operativa que se distribuyó en diferentes períodos, consecutivos entre ellos: el de entrevistas y el de observación. El tiempo requerido para la recopilación completa de datos fue de alrededor de 6 meses.

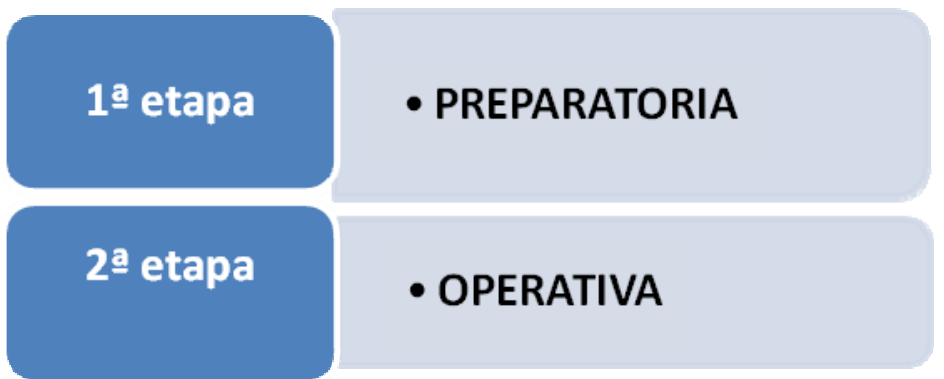

Figura 2. Etapas de trabajo de campo

3 Hemos conservado la denominación original de este documento de planificación y organización para respetar la singularidad de la denominación, si bien interpretado en el contexto educativo genérico debe entenderse en términos genéricos como plan de centro, proyecto de centro, programación general, etc., o incluso en algún caso como España, son todas ellas denominaciones empleadas en las diferentes comunidades autónomas con competencias en materia educativa. 


\section{Resultados}

\section{La planificación y organización de la inclusión en el centro}

En cuanto a la presencia de referencias específicas a medidas organizativas inclusivas en el POF, gran parte de los informantes manifestó desconocerlas, incluyendo entre ellos a los progenitores. Esto es muestra, del lado de los progenitores, que no son conscientes de lo que la institución puede ofrecer a su hijo, así como del lado del profesorado que estos no saben si dichas medidas están o no en el POF. Como el hecho es que sí se recogen medidas organizativas para la inclusión en dicho POF, se deduce que no han leído el POF, que el tema de la inclusión casi nunca se trata y no se tiene en cuenta en la escuela: el POF es un instrumento que es percibido de forma burocrática.

En el POF, demasiado teórico, hay una referencia a la gestión de las intervenciones educativas-docentes, y hay un capítulo dedicado a las personas con discapacidad, aunque de forma "demasiado general, superficial y no específica" (E2DOSOS; E6DOSOS; E8VIDIR; E9DOSOS; E13VIDIR; E14FACIL; E15DOCUR).

En la práctica, la mayoría de los entrevistados transmite tensiones con respecto a la gestión de las intervenciones educativas, proyectando una opinión crítica sobre la falta de especificidad, generalización y escasos detalles sobre las medidas inclusivas en el POF: "En referencia POF se hace en los laboratorios..." (E8VIDIR; E12DOSOS)

Así, en nuestras notas de campo, encontramos opiniones con respecto al POF como: "explica que se llevarán a cabo proyectos educativos, sin definir operativamente... en colaboración con las autoridades locales y familias, pero no dice cómo, o cuando" (O19).

El POF es visto como un documento con mucha teoría y pocas ideas prácticas. La inclusión en el centro, se focaliza en un docente que tiene la función instrumental o de referencia para la inclusión del ACNEE: "hay un profesor que se ocupa de estos temas de inclusión" (E17GENIT).

El Gruppi di Lavoro per l'Handicap d'Istituto (en adelante, GLHI ${ }^{5}$ ), es el grupo de trabajo que se ocupa del Piano Educativo Individualizzato (en adelante, PEI) ${ }^{6}$, pero se opina que no hay coordinación: "las reuniones no se llevan a cabo por falta de personal de $\mathrm{ASL}^{7}$ que no nos apoya" (E1RESOS), o bien, se piensa que "existe el GLHI pero no está en funcionamiento, sólo se ocupa de lo estrictamente necesario" (E3DOSOS; E7FACIL; E9DOSOS; E10DOCUR; E2DOSOS). El GLHI solo se ocupa de la compilación del PEI, que es la herramienta de programación esencial para la asignación a las aulas, pero carente de planificación y actividades curriculares.

Con respecto a la presencia en la escuela de un grupo de trabajo para el ACNEE, la opinión más abundante es que no existe, "aunque hay una existencia considerable de ACNEE en esta escuela no existe" (O2DOCUR; O3), y en la que el director dice: "es un tema nuevo, los

\footnotetext{
4 Los datos textuales literales extraídos de las evidencias empíricas (transcripciones, diarios de campo, etc.) han sido traducidos por los autores del italiano al español.

5 Gruppi di Lavoro per l'Handicap d'Istituto, equivalente al equipo de orientación y apoyo, o los equipos de integración en los centros españoles.

6 Piano Educativo Individualizzato, equivalente a las adaptaciones curriculares individualizadas o a los programas de desarrollo individual en otros contextos.

L'azienda sanitaria locale: institución local de atención sanitaria.

(C) Psy, Soc, \& Educ, 2019, Vol. 11(1)
} 
profesores muestran poco interés en estos asuntos" (E18DIRSC). Esto sugiere que la inclusión no es un objeto de estudio y consideración en esta escuela. En cierto modo, todo queda en el papel, no se ha desarrollado y aplicado: "Honestamente, muchas de estas cosas permanecen sólo en el papel, no se aplican. No hay correspondencia entre lo que está escrito y lo que hace que sea operativa" (E11DOCUR) o "Es un asunto que concierne sólo el maestro de apoyo" (E18DIRSC).

La mayoría de los docentes se expresa negativamente sobre el éxito y el logro de los objetivos establecidos en el POF, en particular con respecto a los alumnos con PEI menos severos, en donde el contenido que hace referencia a las disciplinas se les adapta para llegar a ellos. En cuanto a los alumnos con graves afectaciones llegan a considerar que no tienen herramienta alguna a su disposición: "Estas certificaciones (sic) incluyen el uso de herramientas tecnológicas como soporte y no están allí" (E5DOCUR; E12DOSOS).

\section{Los docentes de apoyo}

Otro tema crítico es el reducido número de profesores de apoyo. La gran cantidad de ACNEE con una presencia escasa de profesorado de apoyo, y un número insuficiente de horas hace que un alumno no pueda ser seguido correctamente y pueda lograr los objetivos destacados en su PEI. Se evidencia falta de comunicación y coordinación efectiva entre los docentes de aula ordinaria y el profesor de apoyo: "no hay coordinación, se evita el probema ignorándolo" (E11DOCUR). La escasez de coordinación entre los profesores de aula ordinaria y profesores de apoyo definen un punto esencial para el fracaso a la hora de alcanzar una organización inclusiva.

El docente de apoyo se expresa de esta manera: "... entre los maestros curriculares tienen dificultades para gestionar su proceso de enseñanza, porque algunos no saben ni la noción de ACNEAE, hay una gran confusión" (E1RESOS). Se abre un surco profundo en una escuela que ve aumentar exponencialmente el número de ACNEAE, con una cantidad disminuida de los docentes de apoyo, unido a una débil formación de los docentes curriculares. Sirva de exponente la decepción presente en los términos expresados por la madre de un estudiante con NEAE asociadas a discapacidad: "creo que lo mejor que puede hacerse para los niños es conocer la discapacidad, no sólo dibujar clasificarlos en una lista, se carece de organización en la selección de los profesores que se asignará a las distintas necesidades los chicos, se hace una asignación arbitraria por la puntuación y sin embargo no es sólo una cuestión de puntuación, es una cuestión de entender los detalles, es esencial"(E17GENIT).

\section{La atención al ACNEAE no diagnosticado}

Una constante en las respuestas de los entrevistados es la negación de la presencia de un equipo docente que se ocupe de los niños que no están diagnósticados, estando ello limitado a la acción formal del GLHI, como órgano específico y semiexterno: "hay reuniones entre los docentes, director, padres y especialistas, hay una discusión entre estos.... pero las reuniones se limitan al GLHI esencialmente" (E1RESOS).

En esencia, los niños con necesidades educativas no diagnósticadas carecen de una atención contingente. La provisión de instrumentos compensatorios se confía a la disponibilidad circunstancial, a pesar de que en las programaciones pueden entrar variantes 
para determinados alumnos (E1RESOS; E6DOSOS; O7; O6). Como no existe un equipo docente responsabilizado de la tarea, un calendario de objetivos a alcanzar y las actividades que deben aplicarse para la inclusión de los alumnos que no tienen diagnóstico, solo las iniciativas individuales de los docentes atienden de forma heterogénea al ACNEAE. Todo está confiado a la buena voluntad y a la sensibilidad de cada profesor: "En cuanto a los instrumentos compensatorios y medidas de apoyo, a menos que estén diagnósticados, sobre qué base se puede poner en práctica una ayuda?" (E11DOCUR). Estas palabras evidencian la discrecionalidad de la intervención del profesorado y la falta de directrices que puedan proporcionar unidad de acción.

No hay interacción entre el GLHI y el claustro. No hay una programación compartida de los objetivos que se persiguen y las actividades que se llevarán a cabo. A pesar de la alta tasa de deserción escolar no se abordan los problemas existentes y las posibles soluciones a adoptar, sobre todo si se tiene en cuenta que: "Esta es una escuela un poco especial, en definitiva, el usuario tiene muchos problemas, algunos socio-culturales, capacidades límite de los maestros curriculares y no" (E6DOSOS ).

\section{La formación y actualización del profesorado}

Cuando se pregunta si la escuela ha proporcionado formación o actualización sobre temas de ACNEAE casi todos responden que no, así como que es escasa la frecuencia con que asiste a actividades de actualización docente, citando razones como: "Mi tasa de reciclaje es baja, debido a que son sólo en la zona...uno tiene que hacerlas por su cuenta e incluso el pago" (E1RESOS; E10DOCUR). U otras más graves como que "no creo que sea cosa útil. No creo que los docentes curriculares deban ser formados, el profesor ya está formado" (E2DOSOS). Otros alegan que se actualizan de forma independiente a través de internet (E3DOSOS) o cuando tienen tiempo disponible (E14FACIL). Aún así, la mitad de ellos reconocen que se actualizan, en promedio, una o dos veces al año (E5DOCUR; E6DOSOS; E7FACIL; E8VIDIR; E11DOCUR; E12DOSOS; E13VIDIR; E15DOCUR).

Resulta relevante la aclaración de un profesor: "una formación y actualizaciones continuas sobre temas de discapacidad, es esencial para hacer un buen trabajo como maestro y se debería ser obligatoria para todos los docentes curriculares" (E6DOSOS). Esta otra visión, minoritaria en nuestro estudio, refleja una conciencia de la necesidad de formación para una escuela inclusiva: "si las partes no tienen la formación, el trabajo no está orientado. La preparación es fundamental, ya que los casos y los problemas son muchos, y cada caso debe aprender" (E3DOSOS).

\section{Discusión y Conclusiones}

Los instrumentos de planificación y organización contienen un proyecto de cambio favorable para la educación del ACNEAE asociadas a discapacidad y a otros factores sociales, en teoría hay un proyecto de inclusión, pero no un compromiso con medidas operativas, a este respecto destacar como se constatan semejanzas respecto a situaciones tanto lejanas en el tiempo y distantes en el espacio (Carrión Martínez, 2001), como otras cercanas (Luque de la Rosa y Carrión Martínez, 2013, 2014; Chiofalo, 2017). 
La escasez de coordinación entre los profesores de aula ordinaria y profesores de apoyo definen un otro punto esencial para explicar un fracaso a la hora de alcanzar una organización inclusiva, que coincide con los resultados de Carrión Martínez, Luque de la Rosa y Fernández Sierra (2013).

En conclusión, desde el punto de vista organizativo el instituto carece de un esqueleto desde el cual comenzar, con claras similitudes con los resultados obtenidos en norte de Italia por Coin (2017). Hay referencias a las prácticas de inclusión en el POF, sobre la gestión de la clase, del tiempo y el espacio, las relaciones entre profesores, alumnos y familias en conexión con lo señalado por Zanobini, Viterbori, Garello y Camba (2017). Pero no encontramos un uso funcional de los recursos profesionales que garantice un proyecto de inclusión transversal.

El problema de la inclusión del alumnado diagnósticado es un problema de muchos jóvenes, los cuales a veces son abandonados a sí mismos como apuntaba Ianes (2009).

Finalmente, en este apartado de conclusiones, para hablar de la lógica inclusiva no se puede ignorar el hecho de que el profesorado debe cuestionar sus acciones si quieren lograr objetivos realistas. Para lograr esto es necesario llevar a cabo una formación básica de todos los docentes, coincidiendo con los resultados de Reina, Hemmelmayr y Sierra-Marroquín (2016), a los que también hay que agregar los gestores y directivos, parte esencial en una perspectiva de mejora y cambio. Es necesario tener profesionales cualificados en este campo, que desempeñen una función de asesoramiento como señalaban Carrión Martinez, Luque de la Rosa y Fernández Sierra (2013), ya que es evidente que no todas las personas involucradas deben tener la misma preparación para hacer frente al problema de la inclusión.

\section{Referencias}

Ainscow, M. (2004). Desarrollo de escuelas inclusivas: Ideas, propuestas y experiencias para mejorar las instituciones escolares. Madrid, España: Narcea.

Albert Gómez, M.J. (2007). La investigación educativa: claves teóricas. Madrid, España: McGraw-Hill.

Avilés Soler, B., Fernández Martínez, M.M., Hernández Garre, C.M., Luque de la Rosa, A. y Gutiérrez Cáceres, R. (2017). Innovaciones para la mejora de la escuela inclusiva: aspectos metodológicos y organizativos. En A. Rodríguez-Martín (Comp.), Prácticas Innovadoras inclusivas: retos y oportunidades (pp. 1377-1383). Oviedo, España: Universidad de Oviedo.

Barton, L. (Comp.) (1988). Discapacidad y sociedad. Madrid, España: Morata.

Barton, L. (Comp.) (2008). Superar las barreras de la discapacidad. Madrid, España: Morata.

Barton, L. y Armstrong, F. (2001). Disability, education and inclusion. Cross cultural issue and dilemmas. In G. Albrecht, K. D. Seelman, \& M. Bury (Ed.), Handbook of Disability Studies (pp. 693-710). Thousand Oaks, CA: Sage.

Carrión Martínez, J.J. (2001). Integración escolar: ¿plataforma para la escuela inclusiva?. Málaga, España: Aljibe.

Carrión Martinez, J. J., Luque de la Rosa, A. y Fernández Sierra, J. (2013). La intervención en audición y lenguaje en los equipos de orientación educativa de Almería (España). Revista Española de Orientación y Psicopedagogía, 24, 42-61.

Cecconi, L. (Ed.). (2002). La ricerca qualitativa in educazione. Milano, Italia: Franco Angeli. 
Chiofalo, T. A. (2017). El fenómeno de la inmigración en la escuela del territorio de Partanna vivido por los docentes. (Tesis doctoral). Universidad de Almería, Almería, España.

Coin, F. (2017). Does Enactive Teaching Improve Inclusion of Immigrant Pupils? A Research in Northern Italy's Schools. SAGE OPEN, 7 (2), 1-12.

D'Alessio, S. (2011a). Decostruire l'integrazione scolastica e costruire l'inclusione in Italia. In R. Medeghini, \& W. Fornasa (Ed.), L'educazione inclusiva. Culture e pratiche nei contesti educativi e scolastici: una prospettiva psicopedgagica (pp. 69-94). Milano, Italia: Franco Angeli.

D'Alessio, S. (2011b). Inclusive education in Italy. A critical analysis of the policy of integrazione scolastica. Rotterdam, Netherlands: Sense Publishers.

D'Alessio, S. (2014). Le normative sui bisogni educativi in Europa e in Italia. Verso un'educazione inclusiva? La prospettiva dei Disability Studies. In P. Gaspari (Ed.), Pedagogia Speciale e BES (pp. 217-244). Roma, Italia: Anicia.

D'Alessio, S. (2015). Commentario a I Bisogni Educativi Speciali: Un'analisi filosofica. In R. Medeghini (Ed.), Norma e Normalità nei Disability Studies (pp. 221-228). Trento, Italia: Erickson.

d'Alonzo, L. (2008). Gestire le integrazioni a scuola. Brescia, Italia: La Scuola.

d'Alonzo, L. (2012). Come fare per gestire la classe nella pratica didattica. Firenze, Italia: Giunti Scuola.

Delli Zotti, G. (2001). Adolescenti tra realtà e costruzione sociale. Gorizia, Eslovenia: Isig.

Dovigo, F. (2007). Fare differenze. Indicatori per l'inclusione scolastica degli alunni con Bisogni Educativi Speciali. Trento, Italia: Erickson.

Echeíta Sarrionandía, G. y Duk Homad, C. (2008). Inclusión educativa. REICE. Revista Iberoamericana sobre Calidad, Eficacia y Cambio en Educación, 6 (2), 1-8.

Echeíta Sarrionandía, G., Simón Rueda, C., Verdugo Alonso, M.A., Sandoval Mena, M., López, M., Calvo Álvarez; M.I. y González Gil, F. (2009). Paradojas y dilemas en el proceso de inclusión educativa en España. Revista de Educación, 349, 153-178.

Flick, U. (2014). El diseño de la Investigación Cualitativa. Madrid, España: Morata.

Gelati, M. (2004). Pedagogia speciale e integrazione. Roma, Italia: Carocci.

Goetz, J.P. y LeCompte, M.D. (2010). Etnografia y diseño cualitativo en investigación educativa. Madrid, España: Morata.

Hierro Parolin, I. C. (2010). L'inclusione scolastica: lo scenario attuale. In I. C. Hierro Parolin (Ed.), Imparare a includere. Riflessioni ed esperienze per una scuola inclusiva (pp.2134). Trento, Italia: Erickson.

Ianes, D. (2009). Qualche spunto di riflessione su integrazione, inclusione, disabilità e Bisogni Educativi Speciali. L'integrazione scolastica e sociale, 8 (5), 444-454.

Ianes, D., Demo, H. y Zambotti, F. (2010). Gli insegnanti e l'integrazione. Atteggiamenti, opinioni e pratiche. Trento, Italia: Erickson.

Lanzara, G. (1988). La progettazione dei sistemi complessi. Saggi, 1, 43-49.

Lucisano, P. y Salerni, A. (2002). Metodologia della ricerca in educazione e formazione. Roma, Italia: Carocci.

Luque de la Rosa, A. y Carrión Martínez, J.J. (2013). Análisis del grado de satisfacción profesional del maestro de audición y lenguaje itinerante. European Journal of Education and Psychology, 6 (1), 55-68. 
Luque de la Rosa, A. y Carrión Martínez, J.J. (2014). Análisis de la actuación docente del maestro de audición y lenguaje en Almería. Revista de Logopedia, Foniatría y Audiología, 34, 68-80. http://dx.doi.org/10.1016/j.rlfa.2013.07.010.

Maviglia, M. (2009). Inclusione e autoanalisi dell'organizzazione scolastica. In R. Medeghini, W. Fornasa, M. Maviglia, \& G. Onger, L'inclusione scolastica. Processi e strumenti di autoanalisi per la qualità inclusiva. Gussago, Italia: Vannini.

Pascale, L., Carrión Martínez, J.J. y Fernández-Martínez, M.M. (2017). El estatus y roles de la familia en la escuela inclusiva en Italia: el caso de un instituto profesional. Revista Nacional e Internacional de Educación Inclusiva, 10 (2), 181-194.

Reina, R., Hemmelmayr, I. y Sierra-Marroquín, B. (2016). Autoeficacia de profesores de educación física para la inclusión de alumnos con discapacidad y su relación con la formación y el contacto previo. Psychology, Society, \& Education, 8(2), 93-103.

Sandri, P. (2014). Integration and inclusion in Italy. Towards a special pedagogy for inclusion Intégration et inclusion en Italie. ALTER, European Journal of Disability Research, 8, 92-104. http://dx.doi.org/10.1016/j.alter.2014.02.004.

Sandrone, G. (2010). L'orientamento e l'integrazione come compiti di sistema. In G. Bertagna, Dirigenti per le scuole. Brescia, Italia: La Scuola.

Santos Guerra, M.A. (2008). Hacer visible lo cotidiano. Madrid, España: Ediciones Akal.

Stake, R. E. (2010). Investigación con estudio de casos. Madrid, España: Morata.

Sturman, A. (1998). Case study Methods. In Education: the complete Encyclopedia. Ámsterdam, Netherlands: Elsevier Science Ltd.

Taylor, S. y Bogdan, R. (2010). Introducción a los métodos cualitativos de investigación. Buenos Aires, Argentina: Paidós.

Tejada Fernández, J. (1997). El proceso de investigación científica. Barcelona, España: Fund. Caixa de Pensions.

Toboso Martín, M., Ferreira, M.A., Díaz Velázquez, E., Fernández-Cid Enríquez; M., Villa Fernández, N. y Gómez de Esteban, C. (2012). Sobre la educación inclusiva en españa: políticas y prácticas. Intersticios: Revista Sociológica de Pensamiento Crítico, 6 (1), 279-295.

Tortora, E. y Santini, R. (2001). Lo studio di caso e il trattamento dei dati delle persone disabili. Recuperado de www.edscuola.it/archivio/handicap/caso.pdf (12-12-2016).

Valdez Contreras, E.F. (2016). Estudio de la formación permanente del profesorado universitario de la Facultad de arquitectura de la Universidad de San Carlos de Guatemala. (Tesis doctoral). Universidad de Almería, Almería, España.

Yin, R.K. (2014). Case study research : design and methods. Los Angeles, USA: Sage Publications Limited.

Zanobini, M., Viterbori, P., Garello, V. y Camba, R. (2017). Parental satisfaction with disabled children's school inclusion in Italy. European Journal of Special Needs Education, pp. 1-18. Article in Press. 